\title{
Method for thermal and structural evaluation of shallow intense-beam deposition in matter
}

\author{
André Pilan Zanoni* \\ CERN, Geneva 23, CH-1211, Switzerland
}

(Received 20 December 2017; published 23 May 2018)

\begin{abstract}
The projected range of high-intensity proton and heavy-ion beams at energies below a few tens of $\mathrm{MeV} / \mathrm{A}$ in matter can be as short as a few micrometers. For the evaluation of temperature and stresses from a shallow beam energy deposition in matter conventional numerical 3D models require minuscule element sizes for acceptable element aspect ratio as well as extremely short time steps for numerical convergence. In order to simulate energy deposition using a manageable number of elements this article presents a method using layered elements. This method is applied to beam stoppers and accidental intense-beam impact onto UHV sector valves. In those cases the thermal results from the new method are congruent to those from conventional solid-element and adiabatic models.
\end{abstract}

DOI: 10.1103/PhysRevAccelBeams.21.054801

\section{INTRODUCTION}

Accelerator facilities broadly employ beam intercepting devices such as beam dumps [1], collimators [2] and beam instruments [3]. For relatively short-ranged interactions such as those of heavy ions [4] or high-intensity particle beam at energies of few MeV/A a tight range profile in the range smaller than a millimeter is observed [5]. The thermal gradients induced by the interaction of a particle beam and matter are simulated through finite element models [6]. To properly characterize shallow thermal gradients conventional models require a huge number of infinitesimal solid elements, tiny time steps and a great amount of computational power.

Tight thermal loading is discussed in [7], which compares the results of a solid, a shell-solid and a multilayered shell model with those of experiments on laser forming of a 2-mm-thick stainless steel plate. Using the multilayered method the simulation efficiency is greatly increased at the expense of a small reduction in accuracy. Another work [8] uses thermomechanical models for multilayered optical coatings in which several layered shells are stacked and coupled to a substrate. However both works do not treat thickness-dependent profiles of heat loading characteristic of beam-energy deposition in matter.

This article introduces a method using multilayered elements to discretize shallow through-thickness heat

\footnotetext{
*andre.pilan.zanoni@cern.ch
}

Published by the American Physical Society under the terms of the Creative Commons Attribution 4.0 International license. Further distribution of this work must maintain attribution to the author(s) and the published article's title, journal citation, and DOI. loading caused by the impact of a particle beam onto a target. We will first apply this method to thin plates of UHV sector valves accidentally hit by a proton beam at $50 \mathrm{MeV}$. Later we will analyze a beam stopper intercepting an ion beam at $72 \mathrm{MeV} / \mathrm{n}$.

\section{MATERIALS AND METHODS}

Consider a target hit by a particle beam (Fig. 1). Using the Monte Carlo particle transport and interaction code FLUKA $[9,10]$ we simulate the interaction between the beam and the target material and determine the energy deposited in the target normalized to one beam particle. For the discretization of the energy deposition the target is divided in energy bins oriented in a Cartesian coordinate system (Fig. 1).

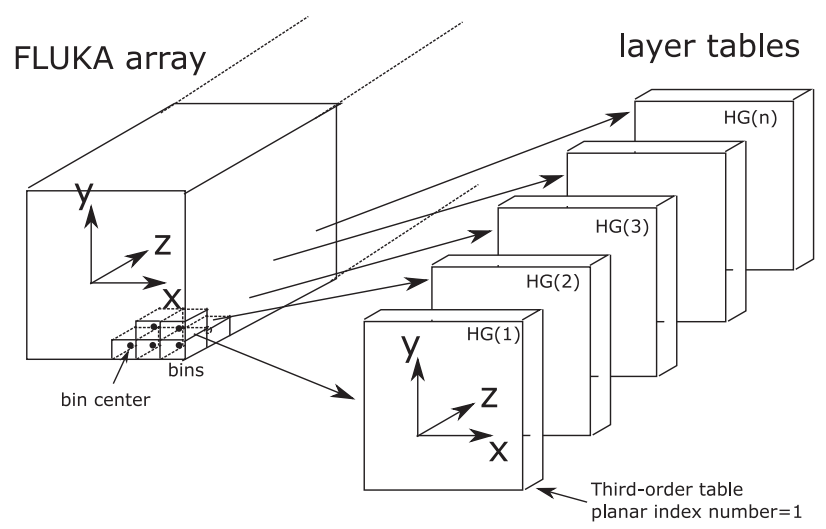

FIG. 1. Schematic segmentation of a data array with energy density values obtained through FLUKA into several table arrays, each with a distinct heat generation profile (HG). The energy density location is applied in the center of the bin. 
An ASCII file outputs the energy generated in each bin. Next we convert the energy density per beam particle $\left(Q_{\mathrm{bp}}\right)$ into power density per pulse $\left(\dot{Q}_{\text {pulse }}\right)$ following Eq. (1):

$$
\dot{Q}_{\text {pulse }}=\frac{Q_{\mathrm{bp}} n_{\mathrm{p}}}{\mu} .
$$

Here $n_{\mathrm{p}}$ is the number of particles per pulse and $\mu$ the beam pulse length.

The power density values are read from the ASCII file and imported in an oriented data table to be applied in an ANSYS 17.1 thermal model [11]. This table contains indexed columns and rows [11] for interpolation of the power density values in solid-element nodes of the thermal model.

For tight geometries accurate finite-element analysis using the full power-density table file requires minuscule solid elements, which is excessively time consuming and computationally demanding.

To treat shallow spatial arrangement we use multilayered elements for geometrical discretization. Note that straightforward nodal interpolation from the full table is not appropriate for a multilayered element as the total thickness is not divided in nodes. Instead we prepare layer-specific heat generation profiles (HG). First we read the ASCII file from FLUKA in a data array which has no indexes and allows data to be read, modified and reshuffled. Next we segment the data array layerwise in third-order tensors with a single planar index number and convert them into powerdensity tables oriented to each layer (Fig. 1).

In ANSYS 17.1 the layered-shell element SHELL131 allows power generation profiles to be input in each layer. SHELL131 is a 3D layered shell element with in-plane and through-thickness thermal conduction capability possessing four nodes, each having up to 32 degrees of freedom (d.o.f.) [11]. For modeling linear through-thickness temperature variation each d.o.f. corresponds to a through-layer temperature [Fig. 2(a)]. In order to reduce the number of integration points and eliminate oscillation between data points we choose the linear temperature variation instead of the quadratic option.

In each shell layer we import the corresponding powerdensity table [Fig. 2(a)]. Segments with relatively small thermal difference along the beam direction are combined into a single layer with linearly averaged power density through the layer thickness.

A thermal model in ANSYS 17.1 allows calculation of the temperature distribution in the target material using Eq. (2):

$$
\rho(T) c_{\mathrm{p}}(T)\left(\frac{\partial T}{\partial t}\right)+\nabla(k(T) T)=\dot{Q}_{\text {pulse }},
$$

where $T$ is the temperature, $t$ the time, $\rho$ the mass density, $c_{\mathrm{p}}$ the specific heat capacity and $k$ the thermal conductivity.

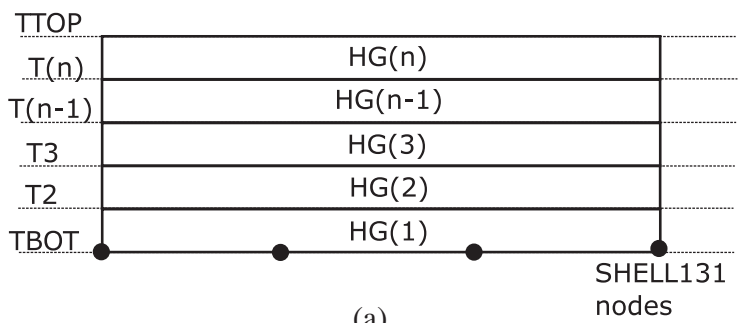

(a)

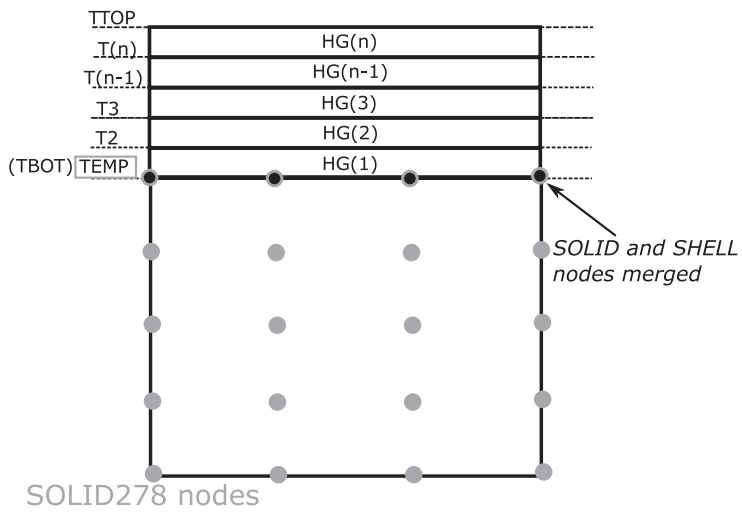

(b)

FIG. 2. (a) Layered shell elements offset to bottom. Each layer has its own heat generation (HG) profile. The temperatures are evaluated as d.o.f. from the shell bottom (TBOT) to the shell top (TTOP). (b) Hybrid model. The shell is offset to bottom. TEMP: d.o.f of a solid-element node.

We implement initial time step lengths smaller than $l^{2} /(4 \alpha)$ [12] where $l$ is the size of the smallest element edge, $\alpha$ the thermal diffusivity rate given by $\alpha=k /\left(\rho c_{p}\right)$ at room temperature.

In order to assess the thermal diffusivity in matter we compare the temperatures obtained through ANSYS 17.1 with adiabatic temperatures. They are calculated from the energy deposition results from FLUKA via Eq. (3) for each bin:

$$
T_{\mathrm{ad}}=\frac{Q_{\mathrm{bp}} n_{\mathrm{p}}}{\bar{\rho} \bar{c}_{\mathrm{p}}}+T_{0}
$$

Here $T_{\mathrm{ad}}$ is the adiabatic temperature, $T_{0}$ the initial temperature, $\bar{\rho}$ the weighted average density and $\bar{c}_{\mathrm{p}}$ the weighted average specific heat capacity between $T_{0}$ and $T_{\text {ad }}$. The weighted averages are evaluated from $T_{0}=20^{\circ} \mathrm{C}$ to $T_{\text {ad }}<600^{\circ} \mathrm{C}$ using intermediate integration points at $100,200,300,400$, and $500^{\circ} \mathrm{C}$.

Maintaining the same multilayered arrangement and time stepping we change the shell element type to SHELL181 for the structural model, which is solved through implicit methods such as $[13,14]$. This element type has four nodes with three translational and three rotational d.o.f. and through-layer integration points [11]. However SHELL181 only calculates in-plane stresses $\left(S_{\mathrm{x}}\right.$, $\left.S_{\mathrm{y}}\right)$ whereas through-thickness stresses are zero $\left(S_{\mathrm{z}}=0\right)$. 
At each time step the layer-specific thermal distributions obtained in the thermal model are imported to the corresponding shell layer in the structural model.

For shallow heat loading in thick bodies we introduce a hybrid shell-solid model [Fig. 2(b)]. The layered-shell nodes are offset to the shell bottom and merged with the top nodes of the solid elements. To model the heat transfer to the solid body we couple the TBOT d.o.f. of the shell to the TEMP d.o.f. of the top nodes of the solid body through a constraint equation [11]. Alternatively the paint application of SHELL131 can be used to replace the TBOT d.o.f. of the shell with the TEMP d.o.f. of the top nodes of the solid body in a thermal simulation [11].

The equivalent stress $\sigma_{\mathrm{e}}$ is calculated through the vonMises yield criterion [15]. Considering $\sigma_{\mathrm{y}}(T)$ the temperature-dependent tensile yield strength a value $\sigma_{\mathrm{e}} / \sigma_{\mathrm{y}}(T)<1$ indicates the deformation induced by thermal gradients is reversible (elastic), otherwise it is permanent (plastic).

\section{RESULTS}

The initial temperature for all models is $22^{\circ} \mathrm{C}$. Neither radiation cooling nor heat transfer through convection is considered.

\section{A. Shallow energy deposition in thin plates}

In the transfer line between Linear accelerator 2 and the Proton Synchrotron Booster at CERN a $50-\mathrm{MeV}$ proton beam pulse could accidentally hit UHV sector valves. The beam pulse is $21.7 \mu$ s long with an intensity of $2.3 \times 10^{13}$ particles and beam sizes at $1 \sigma$ of $\sigma_{\mathrm{h}}=2.9 \mathrm{~mm}, \sigma_{\mathrm{v}}=$ $6.4 \mathrm{~mm}$ as a two-dimensional Gaussian profile [16]. The beam first hits a valve plate and later a counterplate, both 2 $\mathrm{mm}$ thick and with a diameter of $160 \mathrm{~mm}$ (Fig. 3). The combined thickness of both plates is close to the projected range of a $50-\mathrm{MeV}$ proton beam in stainless steel, which is $4.1 \mathrm{~mm}$ [17]. In our geometric model the borders of the plates are simply supported.

The simulation of the beam-matter interaction via FLUKA uses bin sizes of $125 \mu \mathrm{m}$ in the in-plane directions and $40 \mu \mathrm{m}$ through thickness. The cutoff energy is $100 \mathrm{keV}$ for electrons and positrons and $33.3 \mathrm{keV}$ for gamma rays.

We model the properties of stainless steel $316 \mathrm{~L}$ from 20 to $300^{\circ} \mathrm{C}$ [18]: $\rho=7870-7740 \mathrm{~kg} / \mathrm{m}^{3}$, $k=13.9-18.7 \mathrm{~W} /(\mathrm{mK}), \quad c_{\mathrm{p}}=472-512 \mathrm{~J} /(\mathrm{kgK}), \quad \alpha=$ $16.1-17.7 \mu^{\circ} \mathrm{C}^{-1}, \quad E=194-172 \mathrm{GPa}, \quad \nu=0.29-0.31$, $\sigma_{\mathrm{y}}=161-109 \mathrm{MPa}[19,20]$. Here $\alpha$ is the coefficient of thermal expansion, $E$ the Young's modulus and $\nu$ the Poisson's ratio. Temperature-dependent plastic strain is implemented with a multilinear kinematic hardening model using experimental data from [20].

The highest through-thickness thermal difference lies in the beam axis of the counterplate where the maximum difference is about $140{ }^{\circ} \mathrm{C}$ [Fig. 4(a)]. To model this tight energy deposition we compare a thermomechanical model

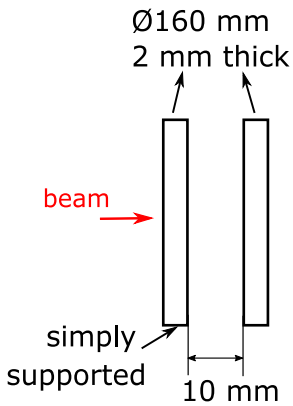

(a)

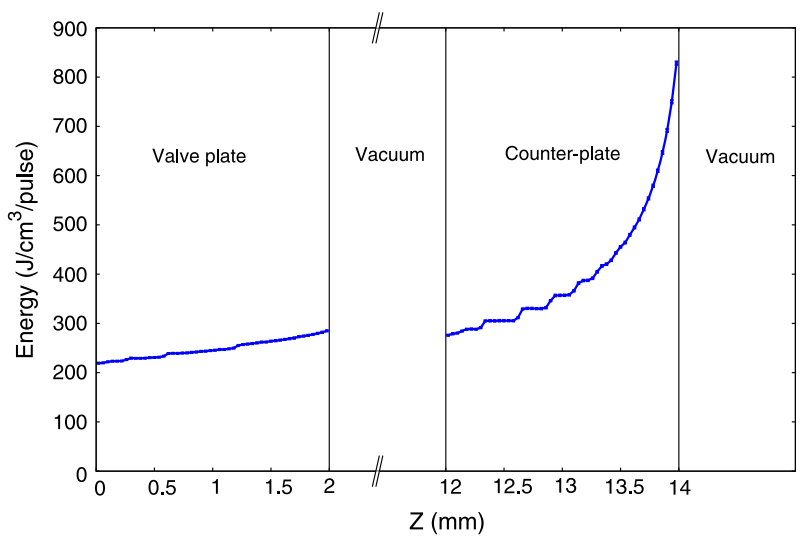

(b)

FIG. 3. (a) Schematic representation of the UHV sector valve. (b) Through-thickness energy deposition in the valve and counterplates along the beam axis after the impact of a proton beam pulse.

of the counterplate with layered-shell elements and another with solid elements [Fig. 4(b)]. We divide the counterplate in 25 layers, each $80 \mu \mathrm{m}$ thick. In the solid-element model the thickness is divided in ten elements.

Both models present congruent through-thickness peak temperature distribution in the beam axis. They match the through-thickness adiabatic profile showing that thermal diffusivity in the counterplate is slow during beam impact.

The maximum equivalent stress lies in the beam axis [Fig. 4(c)] where the counterplate undergoes throughthickness plastic deformation [Fig. 4(d)]. Although the shell-layered model does not compute through-thickness stresses $\left(S_{\mathrm{z}}=0\right)$ both shell-layered and solid models present similar through-thickness distribution of equivalent stress and plastic strain in the beam axis. In fact the maximum through-thickness stress in the solid-element model is very small (max $S_{\mathrm{z}}=0.1 \mathrm{MPa}$ in tension) compared to the maximum in-plane stresses (max $S_{\mathrm{x}}=118 \mathrm{MPa}, \max S_{\mathrm{y}}=157 \mathrm{MPa}$ both in compression). The plate is not constrained in the through-thickness direction being free to expand. The rotational d.o.f. has little influence on the structural results as the maximum rotation angle in the layered-shell model is smaller than $0.1^{\circ}$ while the solid-element model allows no rotation. 


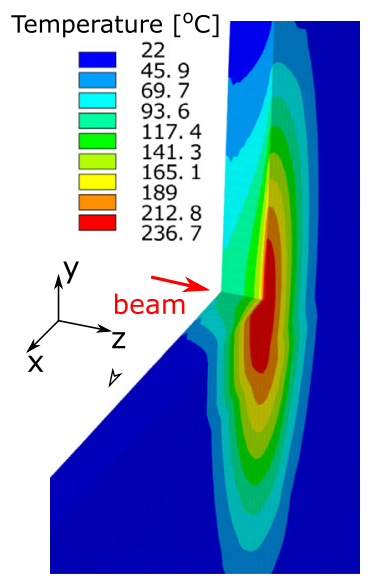

(a)

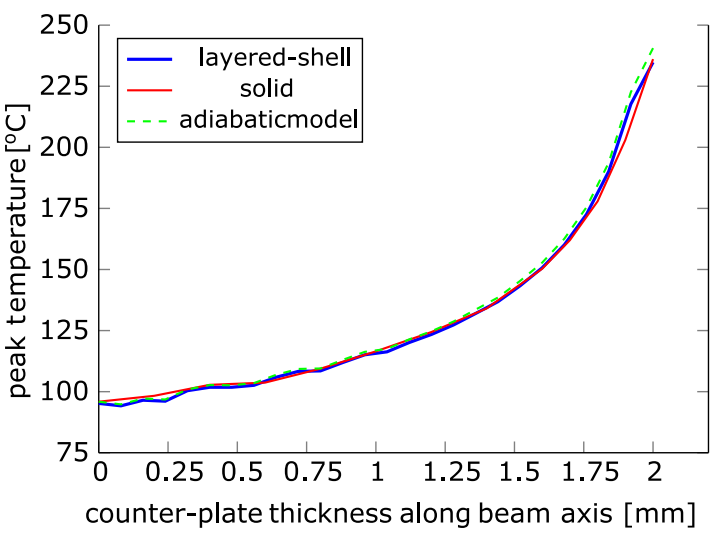

(b)

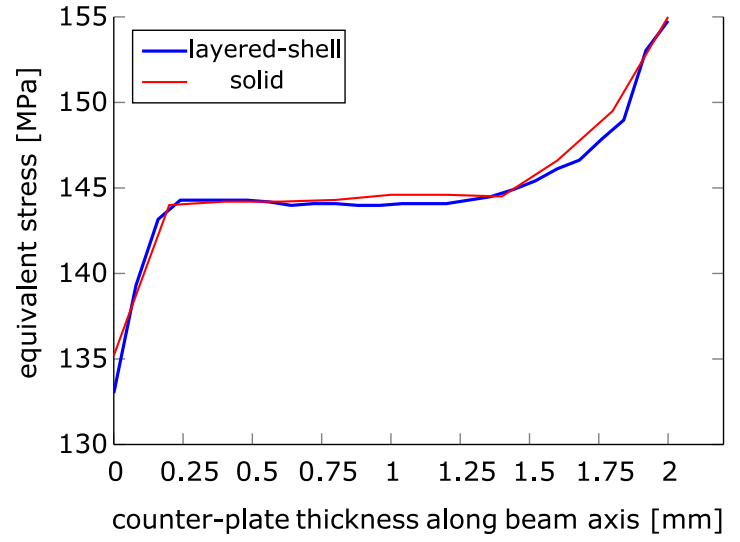

(c)

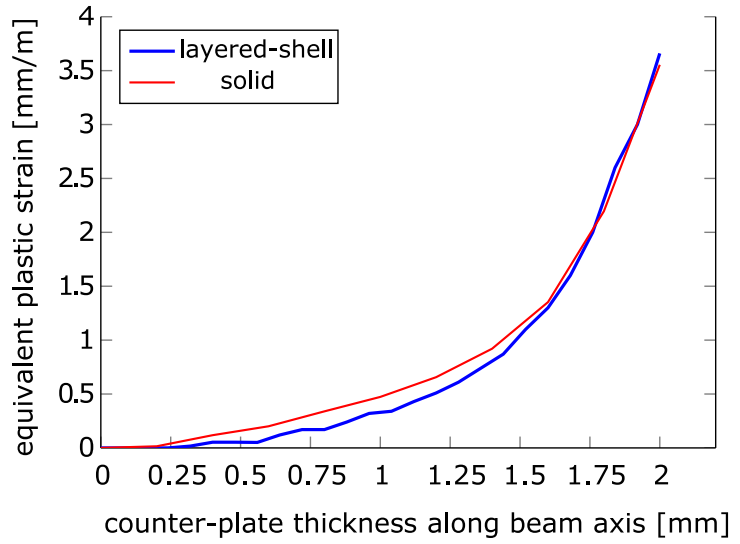

(d)

FIG. 4. (a) Through-thickness temperature distribution in the counterplate at the end of one beam pulse. (b) Peak temperature along the beam axis using a layered-shell model and a solid-element model. (c) Through-thickness equivalent stress and (d) equivalent plastic strain along the beam axis.

Nevertheless the number of elements in the layered-shell model (16352 elements) is much lower than that in the solid-element model (178 800 elements). The simulation elapsed time for both thermal and structural analysis is $58 \mathrm{~min}$ for the layered-shell model and ten hours for the solid-element model.

The layered-shell method can also be applied in cylindrical coordinate systems. Consider a beam stopper out of four concentric graphite shells radially hit by a proton beam pulse at $50 \mathrm{MeV}$ [Fig. 5(a)]. The shells are 170-mm long with outer diameter/thickness of $\varnothing 120 \mathrm{~mm} / 1.6 \mathrm{~mm}, \quad \varnothing 130 \mathrm{~mm} /$ $1.75 \mathrm{~mm}, \varnothing 140 \mathrm{~mm} / 1.85 \mathrm{~mm}$ and $\varnothing 150 \mathrm{~mm} / 2.1 \mathrm{~mm}$. The beam pulse has $120 \mu$ s length and an intensity of $1.27 \times 10^{14}$ protons [21]. The beam sizes at $1 \sigma$ are $\sigma_{\mathrm{h}}=$ $4.4 \mathrm{~mm}$ and $\sigma_{\mathrm{v}}=3.2 \mathrm{~mm}$. In our material model the thermal properties of isostatic graphite from 20 to $600{ }^{\circ} \mathrm{C}$ [18] are $\rho=1900 \mathrm{~kg} / \mathrm{m}^{3}, k=2100-610 \mathrm{~W} /(\mathrm{mK}), c_{\mathrm{p}}=$ 665-1716 J/(kgK). The Bragg peak lies between the two last shells seen by the beam [Fig. 5(b)].

We now return to the energy deposition array. Using cylindrical coordinates we arrange it such that the $\mathrm{Y}$ axis indicates the angle direction, the $\mathrm{Z}$ axis the axial center of the shells and the $\mathrm{X}$ axis the beam axis. The array is sliced in parallel shell layers through the $\mathrm{X}$ axis. We divide each of the two inner shells in two layers and each of the two outer shells in 30 layers.

Figure 5(b) shows the through-thickness temperature profile of the shell-layered model matches the adiabatic temperature profile in the two inner shells. In contrast both profiles are not coincident in the outer shells due to the combination of strong through-thickness thermal gradients and a long beam pulse length (120 $\mu \mathrm{s})$, which allows some thermal diffusion in the beam stopper during beam impact.

\section{B. Shallow energy deposition in thick bodies}

The stopper core present in the extraction line of the Low Energy Ion Ring (LEIR) at CERN is a massive aluminum cylinder with $\varnothing 200 \mathrm{~mm}$ and $210 \mathrm{~mm}$ thickness. The projected range of the lightest extracted ion beam $\left({ }^{4} \mathrm{He}^{2+}\right)$ at $72 \mathrm{MeV} / \mathrm{n}$ in aluminum is $20 \mathrm{~mm}$ [17] meaning this stopper is 10.5 times too long. In copper the projected range falls to $7.5 \mathrm{~mm}$ [17].

LEIR extracts ${ }^{208} \mathrm{~Pb}^{54+}$ ions requiring the beam stopper withstand the impact of one ion beam pulse having 400-ns 


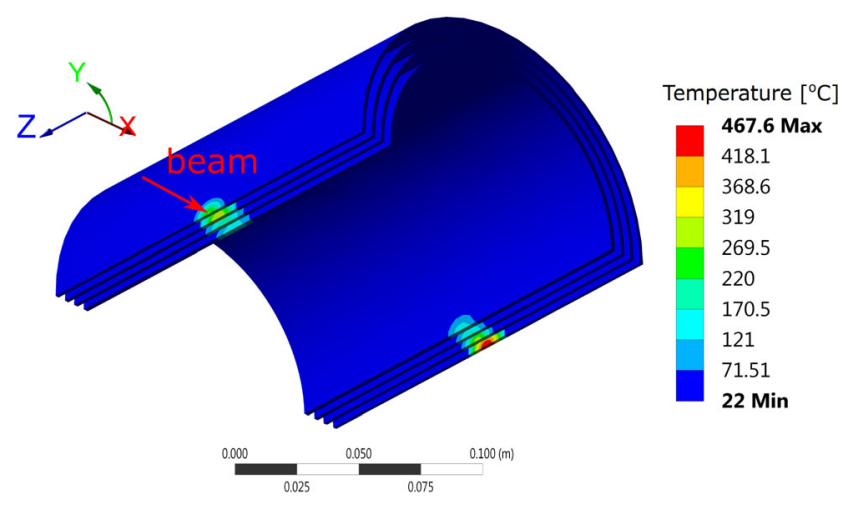

(a)

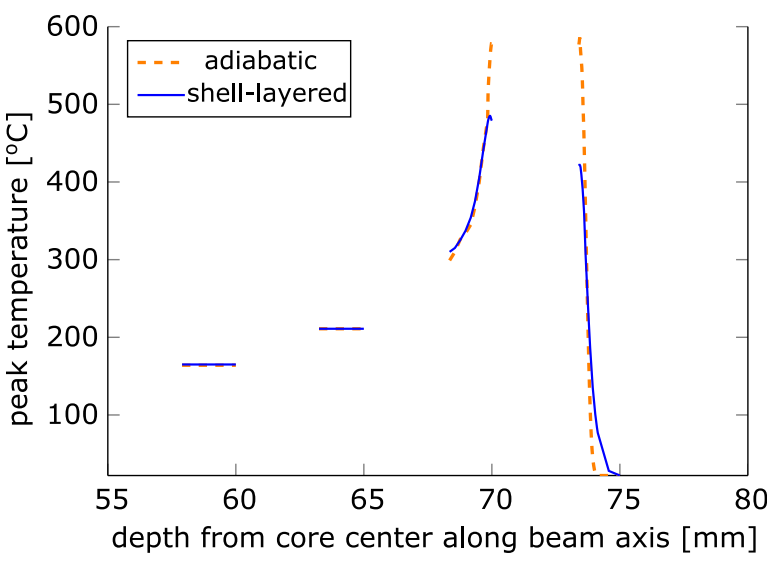

(b)

FIG. 5. (a) Thermal distribution in a beam stopper made of four concentric cylindrical shells out of graphite. The XZ plane is used for symmetry. (b) Peak temperature in the last four shells hit by the beam along the beam axis using a layered-shell model.

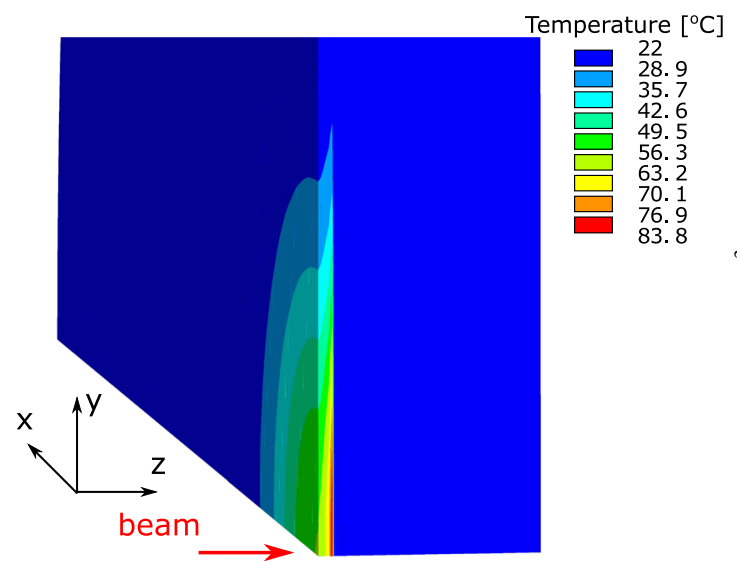

(a)
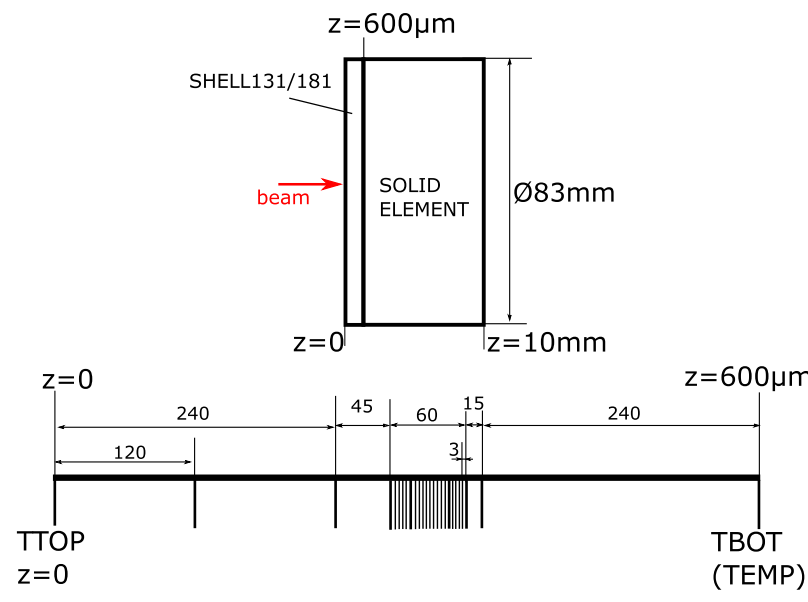

(c)

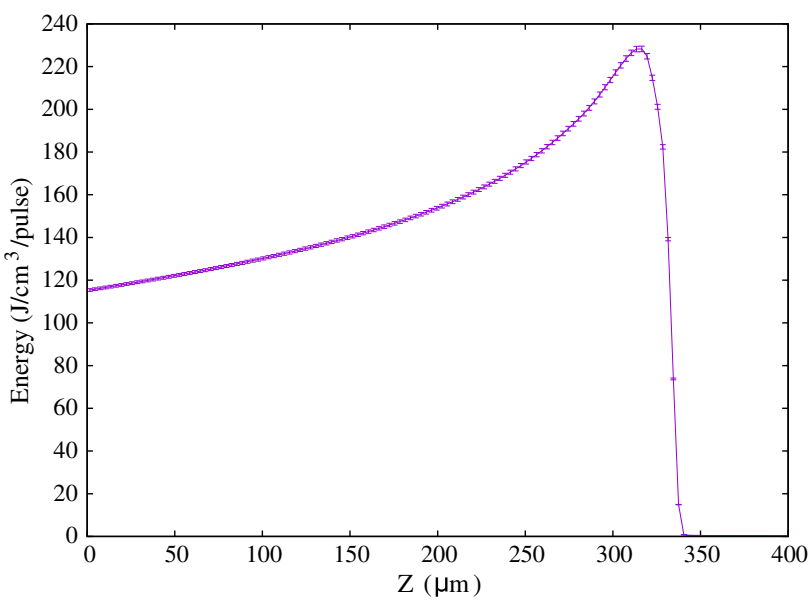

(b)

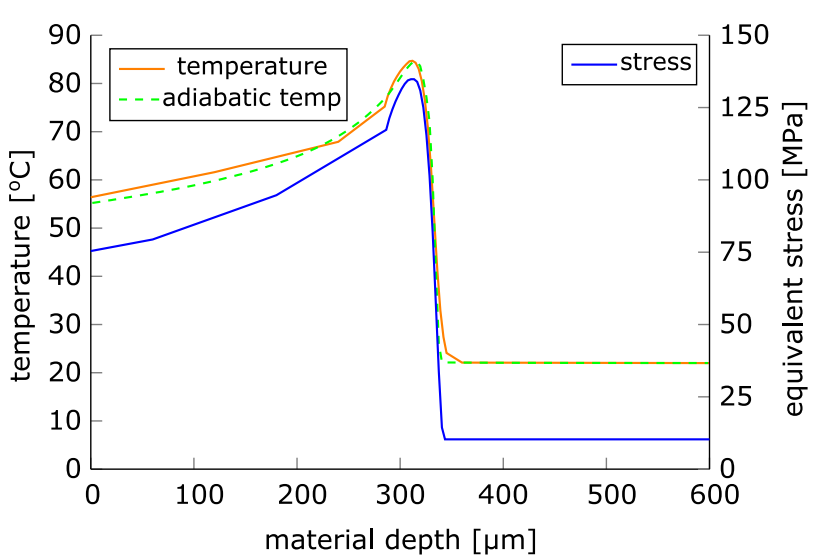

(d)

FIG. 6. (a) Through-thickness thermal distribution in a 10-mm-thick stopper out of the copper alloy CuCr1Zr after one extracted ion beam pulse from LEIR. Two symmetry planes are used. (b) Energy density deposited in the stopper along the beam axis. The energy deposition peaks at $320 \mu \mathrm{m}$. (c) Layer distribution for the thermal and structural analysis. (d) Thermal and in-plane equivalent-stress distribution along the beam axis. 
length, an intensity of $3 \times 10^{9}$ ions and beam sizes at $1 \sigma$ of $\sigma_{\mathrm{h}}=5.0 \mathrm{~mm}$ and $\sigma_{\mathrm{v}}=4.5 \mathrm{~mm} \mathrm{[22].}$

In order to shorten the stopper core we design a $10-\mathrm{mm}$ thick disk [Fig. 6(a)] out of $\mathrm{CuCr} 1 \mathrm{Zr}$, a copper alloy with superior mechanical properties and similar thermal properties as pure copper [23]. The thermal properties of this alloy from 20 to $100{ }^{\circ} \mathrm{C}$ [18] are $\rho=8890-8855 \mathrm{~kg} / \mathrm{m}^{3}$, $k=310-315 \mathrm{~W} /(\mathrm{mK}), \quad c_{\mathrm{p}}=370-450 \mathrm{~J} /(\mathrm{kgK}) \quad[23]$. The bin sizes in FLUKA are $\Delta x=\Delta y=100 \mu \mathrm{m}$ and $\Delta z=3 \mu \mathrm{m}$ for $z<600 \mu \mathrm{m}$. The cutoff energy is $3 \mathrm{keV}$ for electrons and positrons and $1 \mathrm{keV}$ for gamma rays.

We model this disk with a 9.4-mm-thick solid-element body coupled to a $600-\mu \mathrm{m}$-thick shell divided in 22 layers and finely refined around the Bragg peak [Fig. 6(c)]. The shell thickness accommodates all the energy absorbed by the disk from one beam impact as the projected range of an extracted lead ion in the copper alloy is $325 \mu \mathrm{m}$ [17]. The region around the peak energy is strongly discretized with $3-\mu \mathrm{m}$-thick layers.

The highest temperature in the stopper lies in the beam axis. The through-thickness adiabatic temperature profile matches the profile calculated with the hybrid model [Fig. 6(d)]. Due to the short beam pulse length the thermal diffusivity in the beam stopper is slower than the energy deposition during beam impact.

The material is constrained in the in-plane directions while it is free to expand in the through-thickness direction. Under thermal loading the center of the material is strongly compressed in the in-plane directions. The through-thickness stress component is small because the material is free to move in this direction. Knowing that $\max \left|S_{z}\right| \ll$ $\max \left|S_{\mathrm{x}}\right|, \max \left|S_{\mathrm{y}}\right|$ we approximately calculate the maximum equivalent stress at $134 \mathrm{MPa}$ using SHELL181 elements. The maximum equivalent stress is still much lower than the yield strength of $\mathrm{CuCr} 1 \mathrm{Zr}$ at $85^{\circ} \mathrm{C}$ (276.2 MPa) [18]. The maximum rotational angle in the shell is smaller than $0.01^{\circ}$.

The whole hybrid model of the ion stopper uses 14091 elements. In contrast a solid-element model requires 15.0 million elements for uniformly discretizing the vicinity of the beam impact center $\left(\sigma_{\mathrm{h}}=5.0 \mathrm{~mm}, \sigma_{\mathrm{v}}=4.5 \mathrm{~mm}\right.$, range of $325 \mu \mathrm{m}$ ) considering an element size of $9 \mu \mathrm{m} \times$ $9 \mu \mathrm{m} \times 6 \mu \mathrm{m}$ for sufficient thermal resolution around the Bragg peak and good element aspect ratio. This amount of elements is often impracticable using commercial software for finite-element analysis $[11,24]$.

\section{CONCLUSIONS}

This paper presented a method for thermomechanical analysis of shallow energy deposition in targets hit by highintensity particle beam, such as heavy-ion and proton beams below a few tens of MeV/A. Instead of the straightforward but computationally demanding way of using solid elements the method consists of modeling thin material zones with multilayered elements and applying power generation profiles layerwise.

We demonstrated the multilayer technique can be applied to tight energy deposition in thin plates ten times more efficiently than conventional solid-element models with sufficient accuracy for thermal and structural results. For energy deposition in thick bodies in the range of a few hundreds of micrometers we showed the technique allows precise thermal evaluation compared to adiabatic models while fully solid-element models are hardly feasible due to the great number of solid elements required. Designing intercepting devices for high-intensity beams with microscopic projected range in matter is made possible by the method presented in this paper, overcoming the limitation of conventional methods for numerical modeling.

\section{ACKNOWLEDGMENTS}

The author wants to thank Jose Antonio Briz Monago for FLUKA and SRIM calculations and Marco Calviani for support.

[1] A. Perillo-Marcone, A. Sarrio-Martinez, V. Venturi, T. Antonakakis, V. Vlachoudis, E. Nowak, G. Mason, M. Battistin, M. Czapski, and S. Sgobba, Design of air-cooled beam dump for extraction line of PS booster, Conference Proceedings C130512, 4 (2013), THPFI062.

[2] R. W. Assmann et al., The final collimation system for the LHC, Technical Report No. LHC-PROJECT-Report-919, 2006.

[3] P. Strehl, Beam Instrumentation and Diagnostics (Springer, New York, 2006), Vol. 120.

[4] M. Tomut, C. Hubert, W. Mittig, M. Avilov, F. Pellemoine, N. Horny, M. Chirtoc, M. Lang, R. Zabels, and C. Trautmann, Understanding ion-induced radiation damage in target materials, in Proceedings of HB2012, Beijing, China (2012).

[5] J. P. Biersack and J. F. Ziegler, The stopping and range of ions in solids, in Ion Implantation Techniques (Springer, New York, 1982), pp. 122-156.

[6] A. Bertarelli, Beam-induced damage mechanisms, and their calculation, CERN Yellow Reports 2, 159 (2016).

[7] J. Hu, D. Dang, H. Shen, and Z. Zhang, A finite element model using multilayered shell element in laser forming, Opt. Laser Technol. 44, 1148 (2012).

[8] X. heng, L. Zhang, C. Morawe, and M. Sanchez del Rio, Thermal stress prediction in mirror and multilayer coatings, J. Synchrotron Radiat. 22, 317 (2015).

[9] A. Ferrari, P. R. Sala, A. Fasso, and J. Ranft, FLUKA: A multiparticle transport code (Program version 2005), Stanford Linear Accelerator Center Technical Report No. SLAC-R-773, Stanford University, 2005.

[10] T. T. Böhlen, F. Cerutti, M. P. W. Chin, A. Fassò, A. Ferrari, P. G. Ortega, A. Mairani, P. R. Sala, G. Smirnov, and V. Vlachoudis, The FLUKA code: Developments and challenges for high energy and medical applications, Nucl. Data Sheets 120, 211 (2014). 
[11] ANSYS® Academic Research Mechanical, Release 17.1, Ansys Workbench Help.

[12] R. Stout and P. E. David Billings, PEON semiconductor, accuracy and time resolution in thermal transient finite element analysis, in ANSYS-TM Users Conference, 2002.

[13] N. M. Newmark, A method of computation for structural dynamics, J. Eng. Mech. ASCE 85, 67 (1959).

[14] H. M. Hilber, T. J. R. Hughes, and R. L. Talor, Improved numerical dissipation for time integration algorithms in structural dynamics, Earthquake engineering and structural dynamics 5, 283 (1977).

[15] R. v. Mises, Mechanik der festen Körper im plastischdeformablen Zustand, Nach. Gesell. Wiss. Göttingen, Math.-Phys. Kl. 1913, 582 (1913).

[16] A. Pilan Zanoni and J. A. Briz Monago, Thermo-structural analysis on a UHV gate valve (accidental case Linac2/4 to PSB), CERN Technical Report No. PSB-VV-ER-0001, 2017.

[17] J. F. Ziegler, M. D. Ziegler, and J. P. Biersack, SRIM-The stopping and range of ions in matter (2010), Nucl. Instrum. Methods Phys. Res., Sect. B 268, 1818 (2010).

[18] EN-STI-TCD material database, CERN, 2017.
[19] S. Sgobba and F. Leaux, Stainless steel round bars for vacuum applications, CERN Technical Report No. EDMS 790544, 2013.

[20] J. Franklin Dempsey, B. R. Antoun, V. J. Romero, G. W. Wellman, W. M. Scherzinger, and S. Grange, Temperature dependent ductile material failure constitutive modeling with validation experiments, in Challenges in Mechanics of Time-Dependent Materials and Processes in Conventional and Multifunctional Materials (Springer, New York, 2013), Vol. 2, pp. 7-15.

[21] A. Pilan Zanoni and P. García-Ortega, Description and thermo-mechanical analysis of the beam stoppers BI.STPFA and BI.STPSW, CERN Technical Report No. PSB-TSSTP-ES-0003.

[22] R. Scrivens, Beam parameters from Linac 3 to LEIR for beam stoppers, CERN Technical Report No. CPS-TBSEP-0001, 2017.

[23] Deutsches Kupferinstitut, Kupferdatenblatt $\mathrm{CuCr} \mathrm{Cr}$ (Deutsches Kupferinstitut, Düsseldorf, Germany, 2005).

[24] Altair Engineering Inc., HyperWorks 2017 Release Notes, Altair® HyperWorks®. 